Check for updates

Cite this: Mater. Chem. Front. 2020, 4, 3632

Received 1st July 2020,

Accepted 14th August 2020

DOI: $10.1039 / \mathrm{d} 0 \mathrm{qm} 00442 \mathrm{a}$

rsc.li/frontiers-materials

\title{
Doping molecular organic semiconductors by diffusion from the vapor phase $\dagger$
}

\author{
Kelly A. Peterson, (iD a Ashlea Patterson, (iD a Alejandro Vega-Flick, ${ }^{b}$ Bolin Liao ${ }^{b}$ and \\ Michael L. Chabinyc (iD *a
}

\begin{abstract}
Thin films of amorphous small molecule semiconductors are widely used in organic light emitting displays and have promising applications in solar cells and thermoelectric devices. Adding dopants increases the conductivity of organic semiconductors, but high concentrations of dopants can disrupt their structural ordering, alter the shape of the electronic density of states in the material, and increase the effects of Coulomb interactions on charge transport. Electrical doping of the solution processable hole-transport material 2,2',7,7'-tetrakis[N,N-di(4-methoxyphenyl)amino]-9,9'-spirobifluorene (spiro-OMeTAD) was studied with 2,3,5,6-tetrafluoro-7,7,8,8-tetracyanoquinodimethane $\left(F_{4} T C N Q\right)$ as a p-type dopant. Infiltration of $\mathrm{F}_{4} \mathrm{TCNQ}$ from the vapor phase into films of spiro-OMeTAD provided a route to highly doped films with up to $39 \pm 2$ mol\% doping. Structural characterization confirmed that the films remain amorphous even at the highest doping levels with no apparent phase separation. We quantitatively determined the carrier concentration using UV-Vis spectroscopy to interpret the evolution of the electrical conductivity. Over the range of carrier concentrations $\left(10^{19}-10^{20} 1 \mathrm{~cm}^{-3}\right)$, the electrical conductivity increased no more than linearly with carrier concentration, while the thermopower had a small increase with carrier concentration. The trends in conductivity and thermopower were related to the unique electronic structure of spiro-OMeTAD, which is able to support two carriers per molecule. Temperaturedependent conductivity measurements were used to further analyze the transport mechanism.
\end{abstract}

\section{Introduction}

Small molecule organic semiconductors have become widely used in organic light emitting devices (OLEDs) for information displays. ${ }^{1}$ Small molecules can be thermally evaporated to form smooth, glassy layers in devices. Amorphous films of organic semiconductors can also be cast from solvent and are used frequently as electron and hole transport layers in devices such as thin film solar cells. ${ }^{2,3}$ Doping of such transport layers is particularly beneficial to modify their electrical conductivity and to control the energetic line-up of transport levels at interfaces. The role of dopants on the structural ordering of amorphous materials has not been extensively studied, ${ }^{4}$ particularly if there is aggregation induced by charge transfer interactions.

The electrical conductivity of thin films of amorphous small molecules have been found to follow an Arrhenius-type thermally activated hopping conduction model. ${ }^{5}$ The conductivity,

\footnotetext{
${ }^{a}$ Materials Department, University of California, Santa Barbara, California 93106, USA.E-mail: mchabinyc@engineering.ucsb.edu

${ }^{b}$ Department of Mechanical Engineering, University of California, Santa Barbara, California 93106, USA

$\dagger$ Electronic supplementary information (ESI) available. See DOI: 10.1039/ d0qm00442a
}

$\sigma$, is given by eqn (1) where $\sigma_{0}$ is the conductivity at infinite temperature with all carriers activated, $E_{\mathrm{a}}$ is the hopping activation energy, $k_{\mathrm{B}}$ is the Boltzmann constant, and $T$ is the temperature.

$$
\sigma=\sigma_{0} \exp \left(\frac{-E_{\mathrm{a}}}{k_{\mathrm{B}} T}\right)
$$

While eqn (1) describes hopping conduction well, it does not provide any insight into the shape of the electronic density of states (DOS) of the material. The DOS of amorphous molecular semiconductors is usually modeled with a Gaussian form, but the changes in the shape of the DOS with doping are not well understood. Arkhipov et al. proposed a DOS model where a Gaussian distribution of host states is broadened by an exponential tail of trap states. ${ }^{6}$ However, this model is only formulated for dopant concentrations up to $10^{18}-10^{19} \mathrm{~cm}^{-3}$. Recent research on doped small molecule semiconductors has suggested that, at higher dopant concentrations, dopants no longer act as Coulombic traps. ${ }^{7}$ The charge transfer between the host molecules and dopants causes the ionized hosts and dopants to form integer charge transfer complexes (ICTCs) bound with Coulomb forces. This Coulomb interaction modifies the energy level of the ionized host molecules. At $10 \mathrm{~mol} \%$ doping, the concentration of ICTCs is high enough that carriers will 
a

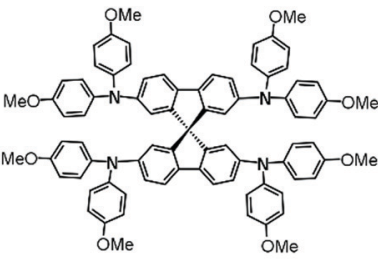

spiro-OMeTAD

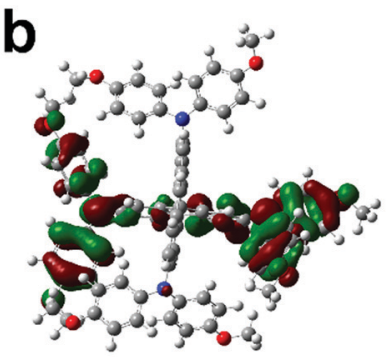

HOMO

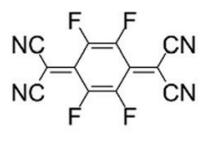

$\mathrm{F}_{4} \mathrm{TCNQ}$

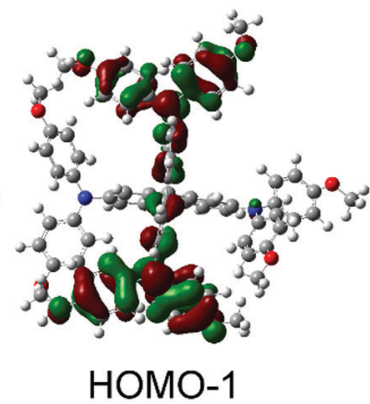

Fig. 1 (a) Chemical structure of spiro-OMeTAD and $F_{4}$ TCNQ and (b) DFT calculations of spiro-OMeTAD's nearly degenerate $\mathrm{HOMO}$ and $\mathrm{HOMO}-1$. Perpendicular fluorene units are both seen edge-on in (b).

preferentially hop among the Coulomb-modified ICTC states. These distributions of ICTC states were described by the width of their static disorder, $\sigma_{\text {ICTC }} \cdot E_{\mathrm{a}}$ was found to be related to both $\sigma_{\text {ICTC }}$ and the relaxation energy of the host molecule after ionization. ${ }^{7}$ Kinetic Monte Carlo simulations have also treated the DOS of small molecule semiconductors as two distributions of states, neutral host states and ionized host states. The conductivity of some small molecule semiconductors increases superlinearly with doping, ${ }^{8,9}$ and these simulations found that a reduction in disorder from adding dopants may explain this superlinear increase. ${ }^{10}$

One example of a solution-processable small molecule semiconductor that forms amorphous films is $2,2^{\prime}, 7,7^{\prime}$-tetrakis $[N, N$ di(4-methoxyphenyl)amino]-9,9'-spirobifluorene (spiro-OMeTAD) (Fig. 1a). This material is commonly used as a hole transport layer in perovskite solar cells ${ }^{3,11}$ and dye sensitized solar cells. ${ }^{12,13}$ In solar cells, spiro-OMeTAD is typically doped with LiTFSI, tert-butylpyridine, and oxygen to a conductivity $\sim 10^{-5} \mathrm{~S} \mathrm{~cm}^{-1}$ at $12-30 \mathrm{~mol} \%$ LiTFSI. $^{14}$ As shown in Fig. 1a, the core of the spiro-OMeTAD molecule has two fluorene units connected by a spiro-bridged carbon atom. Because of this spiro bridge, the two fluorene units are perpendicular to each other, and the molecular structure frustrates crystallization in thin films. Spiro-OMeTAD can be crystallized, leading to a triclinic $P \overline{1}$ structure with only short-range $\pi-\pi$ overlap between adjacent molecules. ${ }^{15,16}$ The spiro-bridge also leads to a unique electronic structure relative to other materials. Density functional theory (DFT) calculations using the long-range corrected hybrid functional $\omega$ B97X-D reveal that the HOMO and HOMO-1 orbitals of spiro-OMeTAD in the experimental geometry from a single crystal structure are nearly degenerate and localize onto opposite sides of the spiro bridge. ${ }^{15,16}$ Our $\omega \mathrm{B} 97 \mathrm{X}-\mathrm{D} /$ 6-31G(d) calculations on an isolated molecule in vacuum without symmetry constraints show similar localization of the
HOMO and HOMO-1 on the asymmetric structure that is likely present in amorphous films (Fig. 1b). Such long-range corrected hybrid DFT functionals are helpful to more accurately model the electronic structure of mixed-valence spiro compounds than functionals such as B3LYP that overly delocalize and mix the molecular orbitals. ${ }^{17}$ The impact of this near degeneracy on charge transport has not been examined in detail.

Here we examine spiro-OMeTAD as a model system to study charge transport in amorphous small molecule semiconductor films at high carrier concentrations. It has the advantage of being readily cast from solution into amorphous thin films and has a unique electronic structure relative to many molecular semiconductors. We doped spiro-OMeTAD films with small molecule dopant 2,3,5,6-tetrafluoro-7,7,8,8-tetracyanoquinodimethane ( $\left.\mathrm{F}_{4} \mathrm{TCNQ}\right)$ by infiltration from the vapor phase leading to a maximum of $39 \pm 2 \mathrm{~mol} \% \mathrm{~F}_{4}$ TCNQ films. The electron affinity of $\mathrm{F}_{4}$ TCNQ at $5.24 \mathrm{eV}^{18}$ is close to Spiro-OMeTAD's first and second ionization energies, $\approx 5.1-5.3 \mathrm{eV} .{ }^{12}$ This close energy match between the dopant and host allowed us to study the effects of spiro-OMeTAD's two hopping sites on the material's electronic transport. We found that Seebeck coefficient and temperature-dependent conductivity measurements are consistent with a Gaussian density of charged states modified by the presence of spiro-OMeTAD ${ }^{2+}$ states.

\section{Results \& discussion}

We doped spun-cast spiro-OMeTAD films by diffusing $\mathrm{F}_{4}$ TCNQ vapor into the films, or "vapor doping" them with $\mathrm{F}_{4}$ TCNQ. We and others have previously used this vapor-doping process to dope semiconducting polymers, including PBTTT and P3HT, ${ }^{19,20}$ but we are not aware of its use for molecular systems. Vapor doping differs from methods, such as thermal co-evaporation of host and dopant molecules or spin casting of doped solutions. Casting the neutral semiconductor prior to the doping process eliminates difficulties in the solubilization of the doped form of the semiconductor and its associated counterion that can preclude achieving high levels of doping. For the vapor doping process, the spiro-OMeTAD films were placed face-down in the top of a doping chamber in a $\mathrm{N}_{2}$ atmosphere glovebox. A small amount of $\mathrm{F}_{4}$ TCNQ was placed in the bottom of the doping chamber. When the doping chamber is heated on a hot plate at $200{ }^{\circ} \mathrm{C}$, the $\mathrm{F}_{4}$ TCNQ sublimates and can diffuse into the film. The glass transition temperature, $T_{\mathrm{g}}$, of spiro-OMeTAD is relatively high at $124{ }^{\circ} \mathrm{C} .{ }^{21}$ In our apparatus, the substrate temperature is not fixed and after $15 \mathrm{~min}$ of heating, the substrate reaches $92.5{ }^{\circ} \mathrm{C}$, and $\sim 106{ }^{\circ} \mathrm{C}$ after 90 min of heating, so $T_{\mathrm{g}}$ of spiro-OMeTAD is never exceeded during the doping process (see ESI $\dagger$ for full heating curve).

We determined whether $\mathrm{F}_{4}$ TCNQ had diffused through the full depth of the film during vapor doping using secondary ion mass spectroscopy (SIMS). Fluorine atoms provide a unique maker for the presence of $\mathrm{F}_{4}$ TCNQ; we note that due to the high fluorine signal level, the amount of $\mathrm{F}_{4}$ TCNQ could not be accurately quantified relative to other atoms using our 


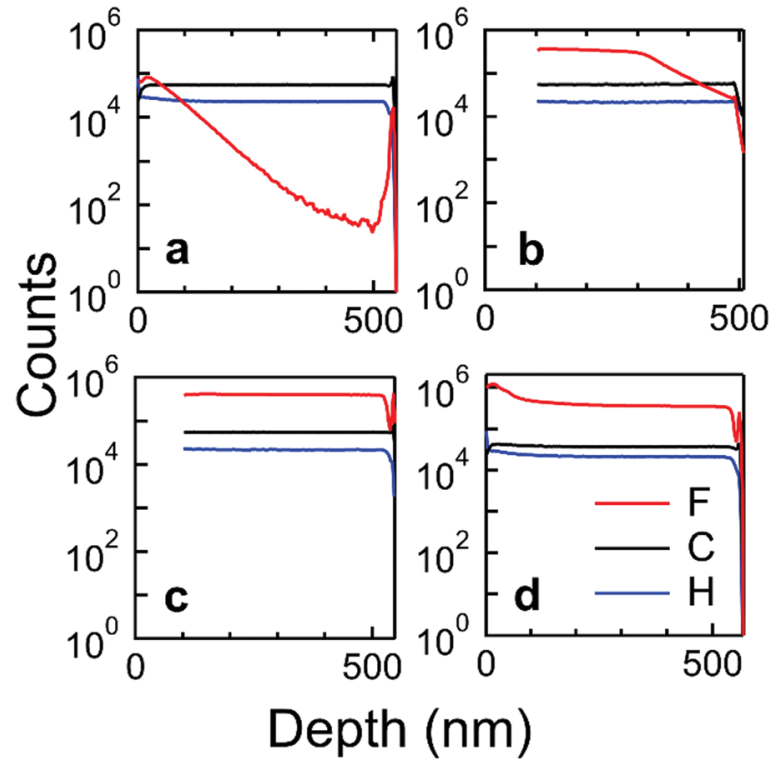

Fig. 2 Secondary ion mass spectroscopy plots tracking the depth distribution of fluorine (red), carbon (black), and hydrogen (blue) in spiroOMeTAD films vapor doped with $\mathrm{F}_{4} \mathrm{TCNQ}$ for (a) $15 \mathrm{~min}$, (b) $30 \mathrm{~min}$, (c) $45 \mathrm{~min}$, and (d) $30 \mathrm{~min}$ with $10 \mathrm{~min}$ annealing. Spikes in the plots at the bottom of the film are an artefact caused by the primary ion beam hitting the insulating quartz substrates.

SIMS instrument. In Fig. 2a, 15 min of vapor doping leads to a concentration profile where the concentration of $\mathrm{F}_{4} \mathrm{TCNQ}$ is greatest near the surface of the film and decreases to the instrument noise level at the bottom of the film. After $30 \mathrm{~min}$ of vapor doping, Fig. 2b, $\mathrm{F}_{4}$ TCNQ has nearly diffused through the full depth of the film. $\mathrm{F}_{4}$ TCNQ can fully diffuse through the film with a roughly even concentration profile after $45 \mathrm{~min}$ of vapor doping, as seen in Fig. 2c. To test whether the uneven concentration profiles caused by vapor doping could be leveled through annealing, we annealed a $30 \mathrm{~min}$ vapor doped film for $10 \mathrm{~min}$ at $140{ }^{\circ} \mathrm{C}$. This annealing temperature was chosen because it is above spiro-OMeTAD's glass transition temperature of $124{ }^{\circ} \mathrm{C}$ but below the temperature at which $\mathrm{F}_{4} \mathrm{TCNQ}$ sublimes from films, $150{ }^{\circ} \mathrm{C} .{ }^{22}$ Fig. $2 \mathrm{~d}$ shows that the concentration profile of the annealed film was more even at the bottom of the film than the unannealed film (Fig. 2b). Ten minutes was long enough for $\mathrm{F}_{4} \mathrm{TCNQ}$ to diffuse through the film without much de-doping but was not long enough for the film to crystallize. In the 45 and 75 min doped films, crystallization was observed around the edges of the film after annealing. Films used for charge transport measurements were annealed to reduce concentration gradients, and partially crystallized areas of annealed films were not measured.

We used grazing incidence wide angle X-ray scattering (GIWAXS) to determine if either spiro-OMeTAD or F4TCNQ crystallized (or co-crystallized) during the doping procedure. In the scattering from the undoped film, Fig. 3a, a broad, nearly isotropic ring of scattering was observed around $q \approx 1.25 \AA^{-1}$. This indicates that the film has an amorphous structure, as expected for spiro-OMeTAD, which is known to generally form
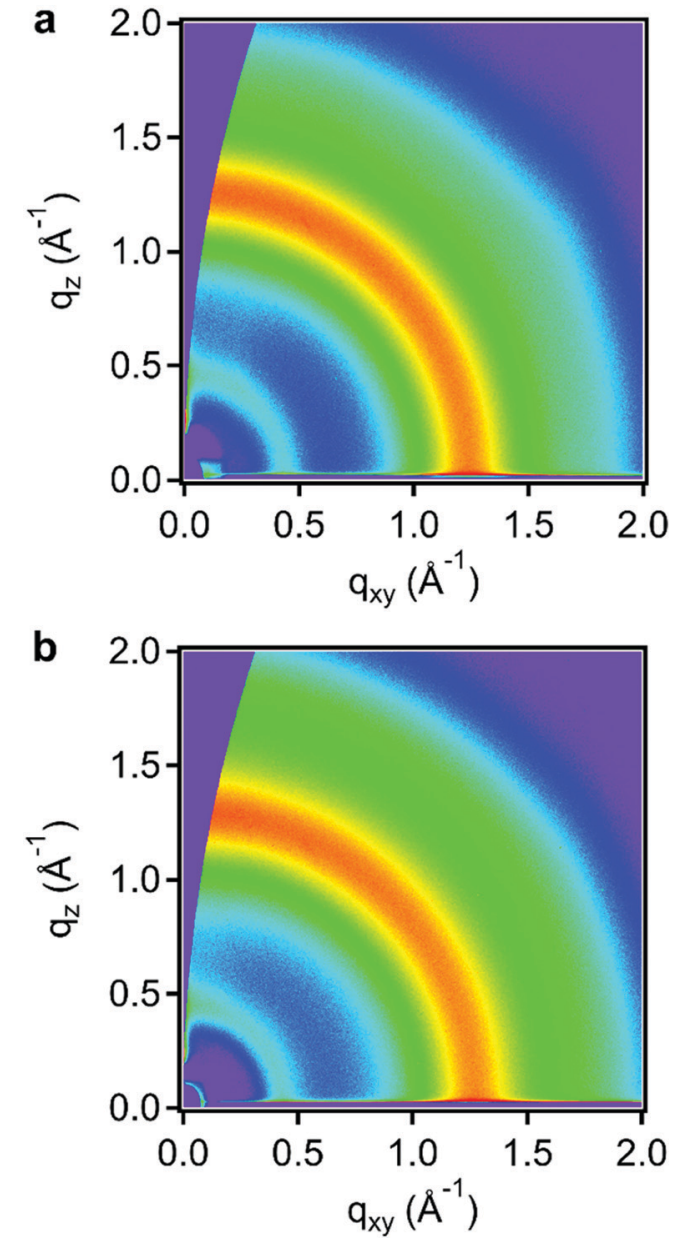

Fig. 3 Grazing incidence wide angle $X$-ray scattering images of (a) neat and (b) 80 min $\mathrm{F}_{4} \mathrm{TCNQ}$ vapor doped spiro-OMeTAD.

glassy films. In Fig. 2b, the scattering for an 80 min $\mathrm{F}_{4} \mathrm{TCNQ}$ vapor doped film showed a similar, nearly isotropic scattering pattern and no signatures of scattering at $d$-spacings known for crystalline form of $\mathrm{F}_{4} \mathrm{TCNQ} .{ }^{23}$ Because this 80 min doping time is higher than the doping times used for charge transport measurements, the X-ray scattering patterns indicate that the doped films retain their amorphous structure throughout the vapor doping process, as opposed to forming a co-crystal or phase separating.

To understand the nature of the charge transfer process between spiro-OMeTAD and $\mathrm{F}_{4} \mathrm{TCNQ}$, we measured the UV-Vis-NIR absorbance as a function of doping time (Fig. 4). The absorbance spectrum of undoped spiro-OMeTAD has an onset around $3 \mathrm{eV}$ with weak sub-optical gap features in the spectrum due to interference effects from the $\sim 500 \mathrm{~nm}$ thick film. Upon doping, new features were observed in the spectra that confirm that integer charge transfer (ICT) occurred between $\mathrm{F}_{4}$ TCNQ and spiro-OMeTAD. Two spectra regions have features that can be assigned to the $\mathrm{F}_{4} \mathrm{TCNQ}$ anion: the vibronic series between $1.3-2 \mathrm{eV}$ and the peak at $2.9 \mathrm{eV} .{ }^{24}$ The features centered around $0.8 \mathrm{eV}$ and $2.5 \mathrm{eV}$ can be assigned to transitions of spiro-OMeTAD ${ }^{+}$ or spiro-OMeTAD ${ }^{2+}$. Spiro-OMeTAD ${ }^{+}$and spiro-OMeTAD ${ }^{2+}$ have 


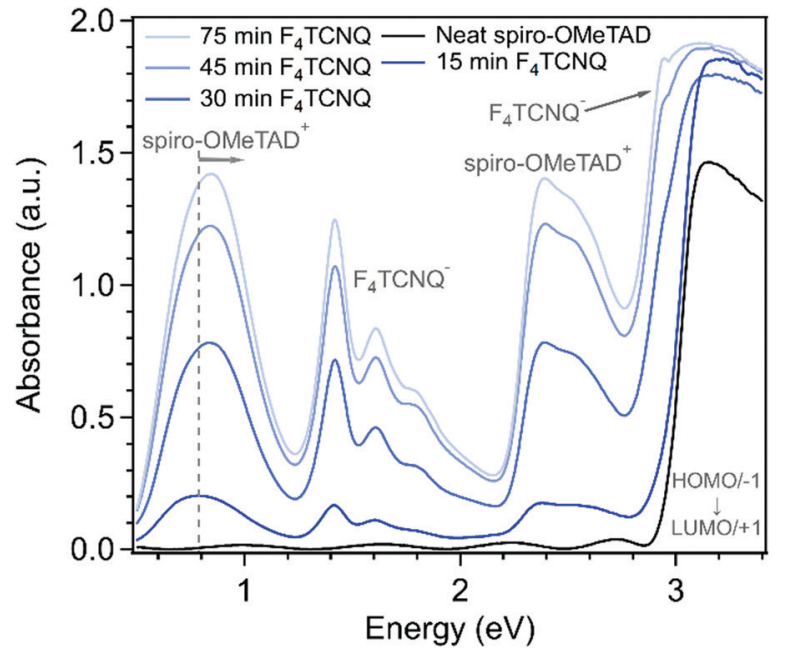

Fig. 4 UV-Vis-NIR spectra of undoped (black) and doped (shades of blue) spiro-OMeTAD films. New peaks in the visible and NIR regions of the doped film spectra indicate the presence of $\mathrm{F}_{4} \mathrm{TCNQ}$ anions and spiro-OMeTAD cations (labeled in gray) and confirm integer charge transfer in the films.

an additional weak transition centered at $1.77 \mathrm{eV}^{25}$ In our spectra, that peak is obscured by the stronger $\mathrm{F}_{4} \mathrm{TCNQ}$ anion absorbance. Due to the degenerate HOMO and HOMO-1 of spiro-OMeTAD, spiro-OMeTAD ${ }^{+}$still has a transition at $3 \mathrm{eV}$. At high doping levels, the feature centered around $0.8 \mathrm{eV}$ becomes less symmetric and shows a blueshift. This blueshift suggests that some population of spiro-OMeTAD molecules has been oxidized to the $2+$ state by two $\mathrm{F}_{4} \mathrm{TCNQ}$ molecules. The energy gap between the HOMO-1 and HOMO levels was calculated to shift from 0.37 to $0.95 \mathrm{eV}$ when spiro-OMeTAD ${ }^{+}$is oxidized to the $2+$ triplet state. ${ }^{26}$ Because spiro-OMeTAD is a mixed-valence compound with two degenerate oxidation states, spiro-OMeTAD ${ }^{2+}$ may be in a triplet state or an open-shell singlet state.

Because spiro-OMeTAD and F4TCNQ undergo integer charge transfer, we used UV-Vis-NIR spectra to estimate the charge carrier concentration in the doped films. In disordered organic semiconductors with hopping conduction, the carrier concentration cannot be measured readily using the Hall effect. Instead, we used the absorbance of $\mathrm{F}_{4} \mathrm{TCNQ}^{-}$to estimate the hole concentration. The area under the $\mathrm{F}_{4} \mathrm{TCNQ}^{-}$peaks between $1.25-2.15 \mathrm{eV}$ is proportional to the concentration of $\mathrm{F}_{4} \mathrm{TCNQ}^{-}$anions in the films. We fit the three $\mathrm{F}_{4} \mathrm{TCNQ}^{-}$peaks and the spiro-OMeTAD ${ }^{+}$transition at $1.77 \mathrm{eV}$ to determine the area of the $\mathrm{F}_{4} \mathrm{TCNQ}^{-}$absorbance. We used reference spectra of $\left[\mathrm{Cp}_{2}{ }_{2} \mathrm{Co}\right]\left[\mathrm{F}_{4} \mathrm{TCNQ}\right]$ and $\left[\mathrm{Cp}^{*}{ }_{2} \mathrm{Fe}\right]\left[\mathrm{F}_{4} \mathrm{TCNQ}\right]$ solutions to calculate a reference value of the molar extinction coefficient, $\varepsilon$ of the $\mathrm{F}_{4} \mathrm{TCNQ}^{-}$optical absorbance. With the $\mathrm{F}_{4} \mathrm{TCNQ}^{-}$absorbance, film thickness, and molar extinction coefficient, the concentration of $\mathrm{F}_{4} \mathrm{TCNQ}^{-}$in each film can be calculated (see ESI, $\dagger$ for full description of fitting methods). The number of $\mathrm{F}_{4} \mathrm{TCNQ}^{-}$is equivalent to the number of electrons removed from spiroOMeTAD and spiro-OMeTAD ${ }^{+}$. The UV-Vis spectra cannot easily be used to distinguish the relative amounts of spiro-OMeTAD ${ }^{+}$ and spiro-OMeTAD ${ }^{2+}$, therefore it is difficult to measure how the holes are distributed on the two possible oxidation sites of a
spiro-OMeTAD molecule. While some spiro-OMeTAD ${ }^{+}$polarons could condense to spiro-OMeTAD ${ }^{2+}$ bipolarons, the shape of the NIR peak suggests that both could be present in the doped films.

On average, films doped to our maximum doping time of $75 \mathrm{~min}$ reached a carrier concentration of $3.3 \pm 0.3 \times 10^{20} \mathrm{~cm}^{-3}$, which is estimated to correspond to a film composition of $39 \pm 2 \mathrm{~mol} \%$ $\mathrm{F}_{4} \mathrm{TCNQ}$ based on the concentration of $\mathrm{F}_{4} \mathrm{TCNQ}^{-}$determined by UV-Vis and the density of spiro-OMeTAD films. ${ }^{27}$ (see ESI $\dagger$ for details) Because the vapor doping process adds mass to the spiro-OMeTAD films, the thickness or density of the films can change. We found that film thicknesses increased from $\approx 420 \pm 20 \mathrm{~nm}$ for undoped films to $\approx 530 \pm 30 \mathrm{~nm}$ for films doped for 45 and $75 \mathrm{~min}$. With this increase in film thickness, the density of spiro-OMeTAD hopping sites would decrease. Based on a spiro-OMeTAD film density of $1.02 \mathrm{~g} \mathrm{~cm}^{-3},{ }^{27}$ the molecule density of spiro-OMeTAD films is $5.02 \times 10^{20} \mathrm{~cm}^{-3}$. Because the HOMO and HOMO-1 of spiro-OMeTAD are nearly degenerate, each molecule has two potential electronic states, giving a hopping site density of $1 \times 10^{21} \mathrm{~cm}^{-3}$.

We measured the room temperature conductivity of several samples with different doping levels. We found that the conductivity generally increases with doping level, with a maximum of $0.017 \mathrm{~S} \mathrm{~cm}^{-1}$ (Fig. 5). This value is two orders of magnitude higher than the conductivity reached by typical LiTFSI doping ${ }^{14}$ and similar to the $0.024 \mathrm{~S} \mathrm{~cm}^{-1}$ reported for LiTFSI and benzoyl peroxide doping. ${ }^{28}$ Estimating with $\sigma=q p \mu$, we found that the electrical mobility may decrease but remains on the order of $10^{-4} \mathrm{~cm}^{2} \mathrm{~V}^{-1} \mathrm{~s}^{-1}$. (see Fig. S8, ESI $\dagger$ ) The mobility of these films cannot be measured directly because they are too disordered for Hall effect measurements, and field-effect transistor and space charge limited current (SCLC) mobility measurements are not accurate on highly doped films. For comparison, the Mott-Gurney SCLC mobility of undoped

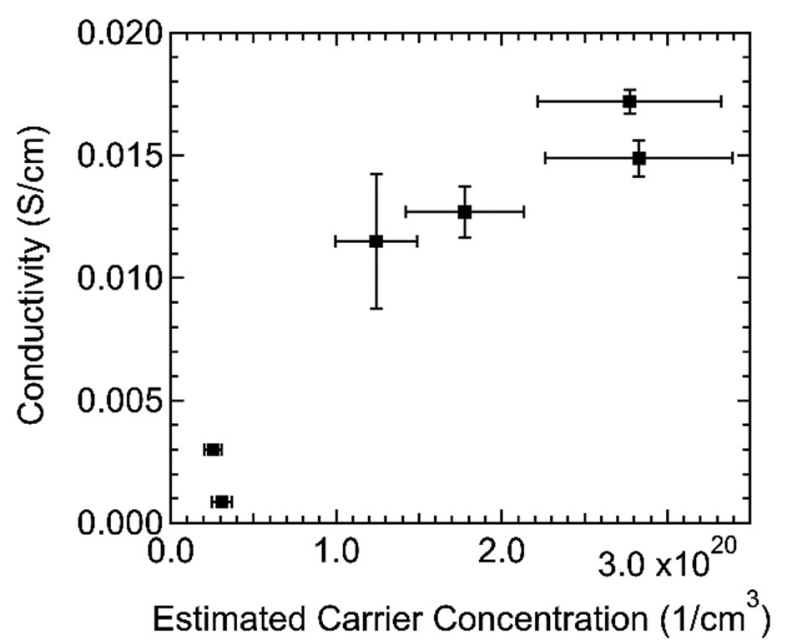

Fig. 5 Room temperature conductivity increases with the estimated carrier concentration in the spiro-OMeTAD: $F_{4} T C N Q$ films. The error in the conductivity is the standard deviation of three measurements taken at different locations of the same film. The error in the carrier concentration is estimated at $20 \%$. 


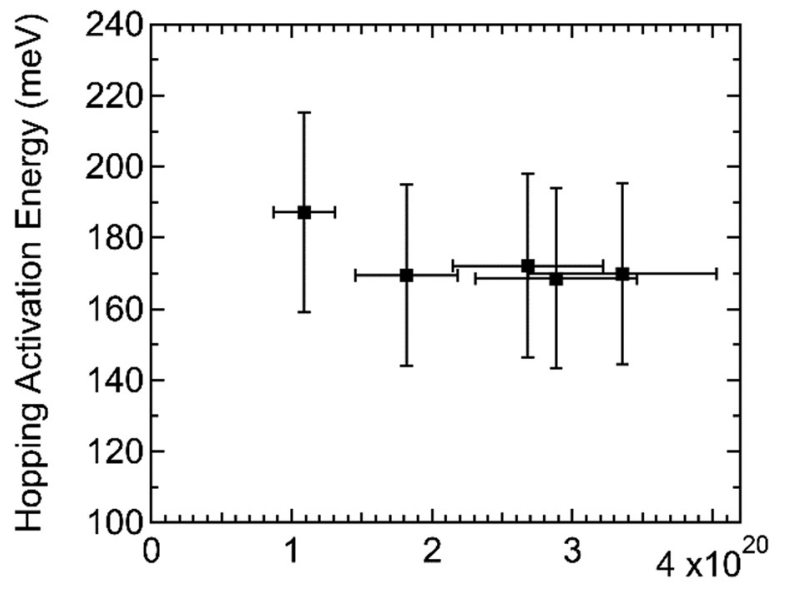

Estimated Carrier Concentration $\left(1 / \mathrm{cm}^{3}\right)$

Fig. 6 Hopping activation energy $\left(E_{\mathrm{a}}\right)$ was roughly constant with carrier concentration in this range. Error in $E_{\mathrm{a}}$ was estimated at $15 \%$, while error in the carrier concentration was estimated at $20 \%$

glassy spiro-OMeTAD was $2.3 \times 10^{-4} \mathrm{~cm}^{2} \mathrm{~V}^{-1} \mathrm{~s}^{-1} \cdot{ }^{29}$ Many doped small molecule systems show a superlinear increase in conductivity with doping, such as MeO-TPD: $\mathrm{F}_{6}$ TCNNQ's increase over a dopant: host density ratio range of $0.01-0.29 .{ }^{9}$ In our measurements of spiro-OMeTAD: $\mathrm{F}_{4} \mathrm{TCNQ}$, the increase in conductivity with hole concentration is no greater than linear, which could be attributed to this decrease in hole mobility.

To find the activation energy of hopping, $E_{\mathrm{a}}$, in this system, we measured the conductivity of films with varying doping levels over a temperature range of at least 140-295 K (see Fig. S9, ESI $\dagger$ ). The dependence of the electrical conductivity on temperature followed the Arrhenius relationship of eqn (1) with a nearly constant $E_{\mathrm{a}} \approx 170 \mathrm{meV}$ (Fig. 6). $E_{\mathrm{a}}$ decreases with increasing doping level and can level off at high doping levels. ${ }^{7}$ The constant (or slightly decreasing) trend of $E_{\mathrm{a}}$ in this carrier concentration range is consistent with $E_{\mathrm{a}}$ leveling off at high doping levels. A broad range of p-type materials has been reported to have $E_{\mathrm{a}}$ values 171-295 $\mathrm{meV}^{7}$ Our measurements of $E_{\mathrm{a}}$ were on films with greater than $10 \mathrm{~mol} \%$ doping, but spiro-OMeTAD: $\mathrm{F}_{4}$ TCNQ is among the systems with lower $E_{\mathrm{a}}$ values. For comparison, 2,2',7,7'-tetrakis( $N, N$-diphenylamino)9.9-spirobifluorene (spiro-TAD): $\mathrm{F}_{6}$ TCNNQ system has similar physical and electronic properties to spiro-OMeTAD: $\mathrm{F}_{4} \mathrm{TCNQ}$ and its $E_{\mathrm{a}}$ is $180 \mathrm{meV}$ at $10 \mathrm{~mol} \%$ doping. ${ }^{30}$ Both spiroTAD: $\mathrm{F}_{6}$ TCNNQ and spiro-OMeTAD: $\mathrm{F}_{4}$ TCNQ have very small host IE-dopant EA offsets $\sim 0.1 \mathrm{eV}$. Interestingly the electrical conductivity of spiro-OMeTAD: $\mathrm{F}_{4}$ TCNQ is significantly higher than that of spiro-TAD: $\mathrm{F}_{6}$ TCNNQ despite the comparable value of $E_{\mathrm{a}}$.

One model proposes that electrical conduction in highly doped small molecule organic semiconductors primarily takes place among host-dopant charge transfer complexes, which form a DOS with width $\sigma_{\text {ICTC. }}{ }^{7}$ Based on data from a wide variety of $\mathrm{p}$ - and n-type host:dopant systems at $10 \mathrm{~mol} \%$ doping, a relationship between $\sigma_{\text {ICTC }}$ determined by ultraviolet photoemission spectroscopy (UPS) and $E_{\mathrm{a}}$, as well as the material's carrier density, was determined. Using the reported empirical relationship between $E_{\mathrm{a}}$ and $\sigma_{\text {ICTC }}$, doped films of spiro-OMeTAD are expected to have a low $\sigma_{\text {ICTC }}$. Spiro-OMeTAD has a low molecular density in doped films of $5 \times 10^{20} \mathrm{~cm}^{-3}$, similar to other materials that are reported to have a low $\sigma_{\text {ICTC }}$. The roughly constant $E_{\mathrm{a}}$ of spiro-OMeTAD:F 4 TCNQ over this doping range suggests that the disorder of integer charge transfer complexes is roughly constant over this doping range, as well.

We measured the in-plane Seebeck coefficient, $S$, of the doped spiro-OMeTAD films with varying carrier concentrations at room temperature to assess how the shape of the electronic DOS changes with doping (Fig. 7a). Because spiro-OMeTAD has two potential sites for charge hopping, it is of interest to determine if there are signs that both oxidation states are involved in charge transport. The Seebeck coefficient is related to the shape of the DOS through the Mott formalism, ${ }^{31}$

$$
S=-\frac{k_{\mathrm{B}}}{e} \int\left(\frac{E-E_{\mathrm{F}}}{k_{\mathrm{B}} T}\right) \frac{\sigma_{\mathrm{E}}}{\sigma}\left(\frac{\partial f}{\partial E}\right) \mathrm{d} E
$$
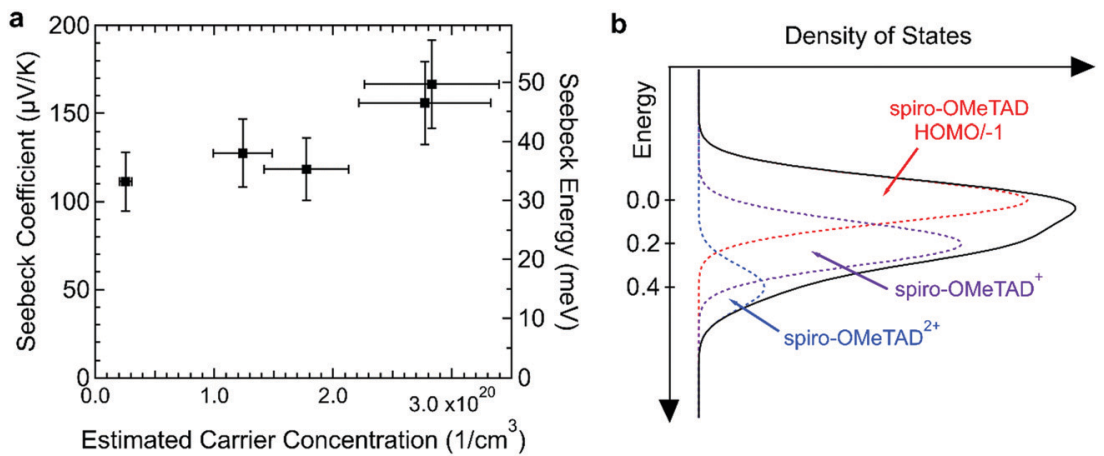

Fig. 7 (a) In-plane Seebeck coefficient was generally constant over this carrier concentration range but increased slightly at $\approx 2.8 \times 10^{20} 1 \mathrm{~cm}^{-3}$. Error in the Seebeck coefficient measurement was estimated at 15\%, while the estimated error in the carrier concentration was $20 \%$. (b) Schematic the electronic DOS of doped spiro-OMeTAD showing the estimated energies to remove an electron from the neutral molecule, spiro-OMeTAD ${ }^{+}$and spiro-OMeTAD ${ }^{2+}$. These states are close enough in energy that the DOS of states is likely broadened by ionized states at high levels of doping. This schematic assumes Gaussian widths of $0.1 \mathrm{eV}$ spaced $0.2 \mathrm{eV}$ apart. 
where $\sigma_{\mathrm{E}}$ is the transport function, $e$ is the electronic charge, $E$ is energy, $E_{\mathrm{F}}$ is the Fermi energy, and $f$ is the Fermi function. In hopping semiconductors, eqn (2) becomes,

$$
S=\frac{k_{\mathrm{B}}}{e}\left(\frac{E_{\mathrm{tr}}-E_{\mathrm{F}}}{k_{\mathrm{B}} T}\right)
$$

where $E_{\mathrm{tr}}$ is the transport energy. In a p-type Gaussian DOS, $E_{\mathrm{tr}}$ is at an energy slightly above the peak of the DOS. The Seebeck coefficient will increase when the distance between $E_{\mathrm{tr}}$ and $E_{\mathrm{F}}$ increases. $^{32}$

We found that $S$ was roughly constant in this carrier concentration range with a small increase at high carrier concentrations $\approx 2.8 \times 10^{20} \mathrm{~cm}^{-3}$. The three points below $2 \times 10^{20} \mathrm{~cm}^{-3}$ have an average value of $119 \mu \mathrm{V} \mathrm{K} \mathrm{K}^{-1}$, while the values at higher concentrations are 156 and $167 \mu \mathrm{V} \mathrm{K} \mathrm{K}^{-1}$. Typically, the Seebeck coefficient of small molecule semiconductors decreases with increasing dopant concentration. ${ }^{9}$ Because the Seebeck constant is related to the distance between $E_{\mathrm{tr}}$ and $E_{\mathrm{F}}$ by eqn (3), $E_{\mathrm{tr}}-E_{\mathrm{F}}$ in spiro-OMeTAD: $\mathrm{F}_{4}$ TCNQ may have increased at $\approx 2.8 \times 10^{20} \mathrm{~cm}^{-3}$. The increase in $E_{\mathrm{tr}}-E_{\mathrm{F}}$ could be caused by a change in DOS shape by spiro-OMeTAD ${ }^{2+}$ states. Upon doping, a Gaussian density of spiro-OMeTAD ${ }^{+}$states forms that overlaps with the Gaussian DOS of spiro-OMeTAD. As the carrier concentration increases, it is possible that bipolarons could form, further extending the tail of the DOS and potentially increasing $E_{\mathrm{tr}}-E_{\mathrm{F}}$. This mechanism of increasing the Seebeck coefficient has been observed and modeled in polymer blends. ${ }^{32,33}$ Fig. 7b shows a schematic of how the densities of charged states could look when the spiro-OMeTAD DOS using three Gaussian functions each with a width of $0.1 \mathrm{eV}$ and with centers spaced $0.2 \mathrm{eV}$ apart. The total number of states used was $1 \times 10^{21} \mathrm{~cm}^{-3}$, with a carrier concentration of $3 \times 10^{20} \mathrm{~cm}^{-3}$ and $25 \%$ of charged molecules in the bipolaron state. These parameters were an approximation based on typical parameters for amorphous small molecules and the properties of $\mathrm{F}_{4}$ TCNQ:spiro-OMeTAD. Differential pulse voltammetry measurements in solvent have shown that the first three oxidation potentials of spiro-OMeTAD are $-5.15,-5.27$, and $-5.48 \mathrm{eV}^{12}$ However, the exact spacing of neutral, 1+, and $2+$ states may change in the solid state, especially if the dielectric constant of the film changes with doping. In addition to changing the shape of the DOS, bipolarons have also been associated with an increase in Seebeck coefficient in hopping semiconductors through an increase in vibrational entropy. ${ }^{34}$

To complement these studies, we studied the changes in thermal transport in spiro-OMeTAD films with doping. We measured the in-plane thermal diffusivity of films with varying carrier concentrations with a transient grating technique. ${ }^{35}$ The transient gradient apparatus uses a visible wavelength laser $(515 \mathrm{~nm}$ or $2.4 \mathrm{eV}$ ) to induce a periodic thermal expansion in the sample. Spiro-OMeTAD ${ }^{+}$and spiro-OMeTAD ${ }^{2+}$ absorb at this wavelength, so the doped films could be measured without any change in sample preparation. Because undoped spiroOMeTAD films are transparent to the pump laser, we prepared a dyed spiro-OMeTAD film by blade-coating a film from a solution of Oil Red $\mathrm{O}$ and spiro-OMeTAD in chlorobenzene. The thermal diffusivity of this pink-dyed film of insulating

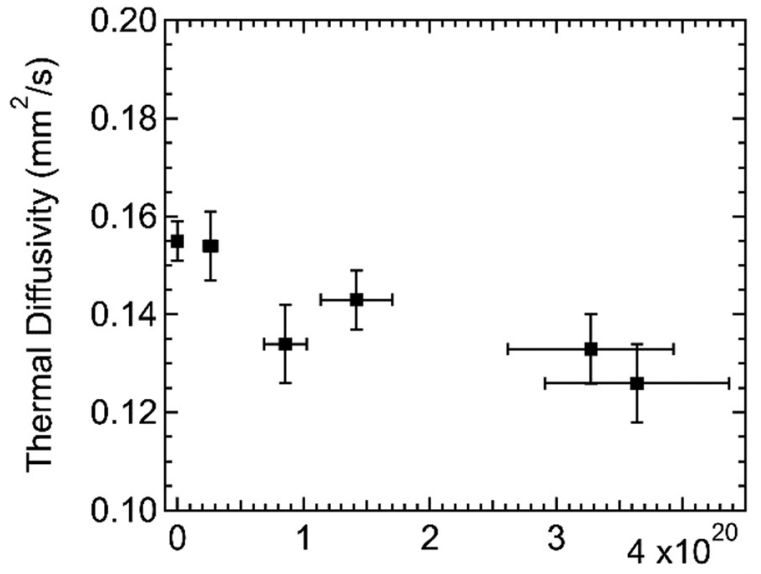

Estimated Carrier Concentration $\left(1 / \mathrm{cm}^{3}\right)$

Fig. 8 In-plane thermal diffusivity of the samples is roughly constant over this carrier concentration range. The $y$ error is the thermal diffusivity fitting error, and the estimated carrier concentration error is $20 \%$.

spiro-OMeTAD was $0.155 \pm 0.004 \mathrm{~mm}^{2} \mathrm{~s}^{-1}$. The thermal diffusivity of $\mathrm{F}_{4} \mathrm{TCNQ}$ vapor-doped films, shown in Fig. 8, was roughly constant, or slightly decreasing, with carrier concentration in the range measured.

The thermal conductivity can be calculated from the product of the thermal diffusivity, density, and specific heat capacity. We measured the specific heat capacity of spiro-OMeTAD powder with modulated differential scanning calorimetry $\left(0.9 \mathrm{~J} \mathrm{~kg}^{-1}{ }^{\circ} \mathrm{C}^{-1}\right)$ and took the density of spiro-OMeTAD films as $1.02 \mathrm{~g} \mathrm{~cm}^{-3} \cdot{ }^{27}$ With these values, we find an in-plane thermal conductivity for spiro-OMeTAD of $0.14 \mathrm{~W} \mathrm{~m}^{-1} \mathrm{~K}^{-1}$. This thermal conductivity is similar to measurements on other amorphous molecules of $0.14-0.15 \mathrm{~W} \mathrm{~m}^{-1} \mathrm{~K}^{-1} \cdot{ }^{36}$ For comparison, undoped $\mathrm{C}_{60}$ and its derivatives have even lower thermal conductivity of $\approx 0.06 \mathrm{~W} \mathrm{~m} \mathrm{~m}^{-1} \mathrm{~K}^{-1,37}$ while undoped films of semiconducting polymers can have thermal conductivities of $\approx 0.2$ to $2 \mathrm{~W} \mathrm{~m} \mathrm{~m}^{-1} \mathrm{~K}^{-1}$ depending on the crystallinity and molecular orientation. ${ }^{38-41}$ The thermal conductivity of doped samples will depend on changes in density or heat capacity, but we can expect that the former is unlikely to increase more than $\approx 20 \%$ (based on the density of crystalline spiro-OMeTAD) and the latter by a comparable factor. Any expected change due to these factors would be relatively small and would tend to bring the thermal conductivity of the doped samples to the same level as the undoped samples. This result is not surprising given the low electrical conductivity of the doped samples but does show that the intermolecular interactions of the charged molecules likely do not substantially modify the thermal conductivity of the amorphous films. The resulting thermoelectric figure of merit is quite low, $Z T=0.8 \times 10^{-4}$ at room temperature.

\section{Conclusions}

We used vapor infiltration to dope spiro-OMeTAD films with $\mathrm{F}_{4}$ TCNQ up to $39 \pm 2 \mathrm{~mol} \% \mathrm{~F}_{4}$ TCNQ. Although spiro-OMeTAD can crystallize in neutral form, the doped films remained 
amorphous. Our results show that this doping method can be used to reach a high conductivity relative to other doped p-type small molecules and provides a convenient route to study doping in small molecules. An increase in the Seebeck coefficient at $\approx 2.8 \times 10^{20} 1 \mathrm{~cm}^{-3}$ suggested that the presence of spiro-OMeTAD ${ }^{2+}$ in the films changes the shape of the DOS. Finally, we found that the thermal diffusivity of spiro-OMeTAD is similar to other amorphous organic materials and does not change significantly with doping. Our findings also suggest that degenerate frontier orbitals may change the thermoelectric properties of organic semiconductors and could be a useful molecular design parameter.

\section{Conflicts of interest}

There are no conflicts of interest to declare.

\section{Acknowledgements}

This work was supported by the National Science Foundation (NSF) under DMR 1808622. Portions of this work were carried out at the MRL Shared Experimental Facilities, supported by the NSF MRSEC program under award no. DMR 1720256. Use of the Stanford Synchrotron Radiation Lightsource, SLAC National Accelerator Laboratory, is supported by the U.S. Department of Energy, Office of Science, Office of Basic Energy Sciences under Contract No. DE-AC02-76SF00515. Use was made of computational facilities purchased with funds from the National Science Foundation (CNS-1725797) and administered by the Center for Scientific Computing (CSC). The CSC is supported by the California NanoSystems Institute (CNSI) and the MRSEC (NSF DMR 1720256) at UC Santa Barbara. We acknowledge the use of the Nanostructures Cleanroom Facility within the CNSI, supported by UC Santa Barbara and the UC Office of the President. A. P. was funded by the FLAM program under NSF award no. DMR 1460656. A. V. and B. L. acknowledges the support of a Sony Faculty Research Award for the thermal transport measurements. We thank Kathryn A. O'Hara and Naveen R. Venkatesan for assistance with GIWAXS measurements and Tom Mates for assistance with SIMS.

\section{References}

1 S. R. Forrest, Organic Electronics: Foundations to Applications, Oxford University Press, Oxford, 2020.

2 L. Calió, S. Kazim, M. Grätzel and S. Ahmad, Hole-Transport Materials for Perovskite Solar Cells, Angew. Chem., Int. Ed., 2016, 55, 14522-14545.

3 J. Urieta-Mora, I. García-Benito, A. Molina-Ontoria and N. Martín, Hole transporting materials for perovskite solar cells: a chemical approach, Chem. Soc. Rev., 2018, 47, 8541-8571.

4 A. P. Proudian, M. B. Jaskot, D. R. Diercks, B. P. Gorman and J. D. Zimmerman, Atom Probe Tomography of Molecular Organic Materials: Sub-Dalton Nanometer-Scale Quantification, Chem. Mater., 2019, 31, 2241-2247.
5 K. Walzer, B. Maennig, M. Pfeiffer and K. Leo, Highly Efficient Organic Devices Based on Electrically Doped Transport Layers, Chem. Rev., 2007, 107, 1233-1271.

6 V. I. Arkhipov, P. Heremans, E. V. Emelianova and H. Bässler, Effect of doping on the density-of-states distribution and carrier hopping in disordered organic semiconductors, Phys. Rev. B: Condens. Matter Mater. Phys., 2005, 71, 045214.

7 M. Schwarze, C. Gaul, R. Scholz, F. Bussolotti, A. Hofacker, K. S. Schellhammer, B. Nell, B. D. Naab, Z. Bao, D. Spoltore, K. Vandewal, J. Widmer, S. Kera, N. Ueno, F. Ortmann and K. Leo, Molecular parameters responsible for thermally activated transport in doped organic semiconductors, Nat. Mater., 2019, 18, 242-248.

8 R. Schmechel, Hopping transport in doped organic semiconductors: A theoretical approach and its application to pdoped zinc-phthalocyanine, J. Appl. Phys., 2003, 93, 4653-4660.

9 T. Menke, D. Ray, H. Kleemann, M. P. Hein, K. Leo and M. Riede, Highly efficient p-dopants in amorphous hosts, Org. Electron., 2014, 15, 365-371.

10 A. Fediai, F. Symalla, P. Friederich and W. Wenzel, Disorder compensation controls doping efficiency in organic semiconductors, Nat. Commun., 2019, 10, 4547.

11 Z. Hawash, L. K. Ono and Y. Qi, Recent Advances in SpiroMeOTAD Hole Transport Material and Its Applications in Organic-Inorganic Halide Perovskite Solar Cells, Adv. Mater. Interfaces, 2018, 5, 1700623.

12 W. H. Nguyen, C. D. Bailie, E. L. Unger and M. D. McGehee, Enhancing the Hole-Conductivity of Spiro-OMeTAD without Oxygen or Lithium Salts by Using Spiro(TFSI)2 in Perovskite and Dye-Sensitized Solar Cells, J. Am. Chem. Soc., 2014, 136, 10996-11001.

13 T. Leijtens, J. Lim, J. Teuscher, T. Park and H. J. Snaith, Charge Density Dependent Mobility of Organic HoleTransporters and Mesoporous TiO2 Determined by Transient Mobility Spectroscopy: Implications to Dye-Sensitized and Organic Solar Cells, Adv. Mater., 2013, 25, 3227-3233.

14 A. Abate, T. Leijtens, S. Pathak, J. Teuscher, R. Avolio, M. E. Errico, J. Kirkpatrik, J. M. Ball, P. Docampo, I. McPherson and H. J. Snaith, Lithium salts as "redox active" p-type dopants for organic semiconductors and their impact in solid-state dye -sensitized solar cells, Phys. Chem. Chem. Phys., 2013, 15, 2572-2579.

15 D. Shi, X. Qin, Y. Li, Y. He, C. Zhong, J. Pan, H. Dong, W. Xu, T. Li, W. Hu, J.-L. Brédas and O. M. Bakr, Spiro-OMeTAD single crystals: Remarkably enhanced charge-carrier transport via mesoscale ordering, Sci. Adv., 2016, 2, e1501491.

16 Y. Li, H. Li, C. Zhong, G. Sini and J.-L. Brédas, Characterization of intrinsic hole transport in single-crystal spiroOMeTAD, npj Flexible Electron., 2017, 1, 2.

17 T. Körzdörfer and J.-L. Brédas, Organic Electronic Materials: Recent Advances in the DFT Description of the Ground and Excited States Using Tuned Range-Separated Hybrid Functionals, Acc. Chem. Res., 2014, 47, 3284-3291.

$18 \mathrm{~W}$. Gao and A. Kahn, Controlled p-doping of zinc phthalocyanine by coevaporation with tetrafluorotetracyanoquinodimethane: A direct and inverse photoemission study, Appl. Phys. Lett., 2001, 79, 4040-4042. 
19 S. N. Patel, A. M. Glaudell, K. A. Peterson, E. M. Thomas, K. A. O'Hara, E. Lim and M. L. Chabinyc, Morphology controls the thermoelectric power factor of a doped semiconducting polymer, Sci. Adv., 2017, 3, e1700434.

20 E. Lim, K. A. Peterson, G. M. Su and M. L. Chabinyc, Thermoelectric Properties of Poly(3-hexylthiophene) (P3HT) Doped with 2,3,5,6-Tetrafluoro-7,7,8,8-tetracyanoquinodimethane (F4TCNQ) by Vapor-Phase Infiltration, Chem. Mater., 2018, 30, 998-1010.

21 T. Malinauskas, D. Tomkute-Luksiene, R. Sens, M. Daskeviciene, R. Send, H. Wonneberger, V. Jankauskas, I. Bruder and V. Getautis, Enhancing Thermal Stability and Lifetime of SolidState Dye-Sensitized Solar Cells via Molecular Engineering of the Hole-Transporting Material Spiro-OMeTAD, ACS Appl. Mater. Interfaces, 2015, 7, 11107-11116.

22 J. Li, C. W. Rochester, I. E. Jacobs, S. Friedrich, P. Stroeve, M. Riede and A. J. Moulé, Measurement of Small Molecular Dopant F4TCNQ and C60F36 Diffusion in Organic Bilayer Architectures, ACS Appl. Mater. Interfaces, 2015, 7, 28420-28428.

23 J. E. Cochran, M. J. N. Junk, A. M. Glaudell, P. L. Miller, J. S. Cowart, M. F. Toney, C. J. Hawker, B. F. Chmelka and M. L. Chabinyc, Molecular Interactions and Ordering in Electrically Doped Polymers: Blends of PBTTT and F4TCNQ, Macromolecules, 2014, 47, 6836-6846.

24 D. A. Dixon, J. C. Calabrese and J. S. Miller, Crystal and molecular structure of the 2:1 charge-transfer salt of decamethylferrocene and perfluoro-7,7,8,8-tetracyano-pquinodimethane: $\left[\left[\mathrm{Fe}\left(\mathrm{C}_{5} \mathrm{Me}_{5}\right)_{2}\right]+\text {.cntdot. }\right]_{2}[\mathrm{TCNQF} 4]_{2}{ }^{-}$. The electronic structure of $\left[\mathrm{TCNQF}_{4}\right]_{n}(n=0,1-, 2-), J$. Phys. Chem., 1989, 93, 2284-2291.

25 U. Bach, PhD thesis, École Polytechnique Fédérale de Lausanne, 2000.

26 S. Fantacci, F. De Angelis, M. K. Nazeeruddin and M. Grätzel, Electronic and Optical Properties of the SpiroMeOTAD Hole Conductor in Its Neutral and Oxidized Forms: A DFT/TDDFT Investigation, J. Phys. Chem. C, 2011, 115, 23126-23133.

27 P. Docampo, A. Hey, S. Guldin, R. Gunning, U. Steiner and H. J. Snaith, Pore Filling of Spiro-OMeTAD in Solid-State Dye-Sensitized Solar Cells Determined Via Optical Reflectometry, Adv. Funct. Mater., 2012, 22, 5010-5019.

28 Q. Liu, L. Fan, Q. Zhang, A. Zhou, B. Wang, H. Bai, Q. Tian, B. Fan and T. Zhang, Benzoyl Peroxide as an Efficient Dopant for Spiro-OMeTAD in Perovskite Solar Cells, ChemSusChem, 2017, 10, 3098-3104.
29 J. A. Röhr, X. Shi, S. A. Haque, T. Kirchartz and J. Nelson, Charge Transport in Spiro-OMeTAD Investigated through Space-Charge-Limited Current Measurements, Phys. Rev. Appl., 2018, 9, 044017.

30 F. Zhang and A. Kahn, Investigation of the High Electron Affinity Molecular Dopant F6-TCNNQ for Hole-Transport Materials, Adv. Funct. Mater., 2017, 1703780.

31 N. F. Mott and E. A. Davis, Electronic processes in noncrystalline materials, Oxford University Press, Oxford, 2nd edn, 1979.

32 G. Zuo, X. Liu, M. Fahlman and M. Kemerink, High Seebeck Coefficient in Mixtures of Conjugated Polymers, Adv. Funct. Mater., 2017, 1703280.

33 A. Abtahi, S. Johnson, S. M. Park, X. Luo, Z. Liang, J. Mei and K. R. Graham, Designing $\pi$-conjugated polymer blends with improved thermoelectric power factors, J. Mater. Chem. A, 2019, 7, 19774-19785.

34 D. Emin, Enhanced Seebeck coefficient from carrierinduced vibrational softening, Phys. Rev. B: Condens. Matter Mater. Phys., 1999, 59, 6205-6210.

35 A. Vega-Flick, D. Jung, S. Yue, J. E. Bowers and B. Liao, Reduced thermal conductivity of epitaxial GaAs on Si due to symmetry-breaking biaxial strain, Phys. Rev. Mater., 2019, 3, 034603.

36 X. Xie, K. Yang, D. Li, T.-H. Tsai, J. Shin, P. V. Braun and D. G. Cahill, High and low thermal conductivity of amorphous macromolecules, Phys. Rev. B, 2017, 95, 035406.

37 X. Wang, C. D. Liman, N. D. Treat, M. L. Chabinyc and D. G. Cahill, Ultralow thermal conductivity of fullerene derivatives, Phys. Rev. B: Condens. Matter Mater. Phys., 2013, 88, 075310.

38 J. C. Duda, P. E. Hopkins, Y. Shen and M. C. Gupta, Thermal transport in organic semiconducting polymers, Appl. Phys. Lett., 2013, 102, 251912.

39 H. Ushirokita and H. Tada, In-plane Thermal Conductivity Measurement of Conjugated Polymer Films by Membranebased AC Calorimetry, Chem. Lett., 2016, 45, 735-737.

40 J. Liu, X. Wang, D. Li, N. E. Coates, R. A. Segalman and D. G. Cahill, Thermal Conductivity and Elastic Constants of PEDOT:PSS with High Electrical Conductivity, Macromolecules, 2015, 48, 585-591.

41 A. Roy, T. L. Bougher, R. Geng, Y. Ke, J. Locklin and B. A. Cola, Thermal Conductance of Poly(3-methylthiophene) Brushes, ACS Appl. Mater. Interfaces, 2016, 8, 25578-25585. 